https://doi.org/10.35336/VA-2020-E-3-9

\title{
REMOTE MONITORING FOR THE EARLY DETECTION OF CHANGES IN PATIENT STATUS USING THE HOME MONITORING TECHNOLOGY
}

A.Sh.Revishvili ${ }^{1}$, N.N.Lomidze ${ }^{1}$, A.S.Abdrakhmanov ${ }^{2}$, A.A.Nechepurenko ${ }^{3}$, E.A.Ivanitsky ${ }^{4}$, O.V.Belyaev ${ }^{5}$, S.V.Popov ${ }^{6}$, D.S.Lebedev ${ }^{7}$, V.K.Lebedeva ${ }^{7}$, S.P.Mikhailov ${ }^{8}$, E.A.Pokushalov ${ }^{9}$, S.E.Mamchur ${ }^{10}$, P.L.Shugaev ${ }^{11}$, R.R.Rekvava ${ }^{12}$, S.N.Vasilyev ${ }^{13}$, V.V.Kuptsov ${ }^{1}$, V.I.Berdyshev ${ }^{13}$, R.Sh.Sungatov ${ }^{14}$, I.Sh.Khassanov ${ }^{15,16}$ on behalf of the ReHoming study investigators

${ }^{1}$ Visnevsky National Medical Research Center of Surgery, Russian Ministry of Healthcare, Moscow, Russia, ${ }^{2}$ National Scientific Cardiosurgical Center, Nur-Sultan, Kazakhstan, ${ }^{3}$ Federal Center of Cardiovascular Surgery, Astrakhan, Russia, ${ }^{4}$ Federal Center of Cardiovascular Surgery, Krasnoyarsk, Russia, ${ }^{5}$ Sverdlovsk Regional Clinical Psychoneurological Hospital for War Veterans, Ekaterinburg, Russia, ${ }^{6}$ Scientific Research Institute of Cardiology, Tomsk National Research Medical Center of Russian Academy of Sciences, Tomsk, Russia, ${ }^{7}$ Almazov National Medical Research Center, Russian Ministry of Healthcare, Saint Petersburg, Russia, ${ }^{8}$ Sverdlovsk Regional Clinical Hospital \#1, Ekaterinburg, Russia, ${ }^{9}$ Meshalkin National Medical Research Center, Russian Ministry of Healthcare, Novosibirsk, Russia, ${ }^{10}$ Scientific Research Institute for Complex Problems of Cardiovascular Diseases of Siberian Branch

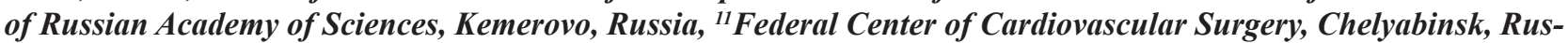
sia, ${ }^{12}$ Scientific Research Institute of Cardiology and Internal Diseases, Ministry of Healthcare, Almaty, Kazakhstan,

${ }^{13}$ Institute of Mathematics and Mechanics, Ural Branch of Russian Academy of Sciences, Ekaterinburg, Russia, ${ }^{14}$ Dicom Consulting LLC, Kazan, Russia, ${ }^{15}$ BIOTRONIK, Berlin, Germany, ${ }^{16}$ Max Schaldach-Stiftungsprofessur für Biomedizinische Technik, Friedrich-Alexander University Erlangen-Nuremberg, Erlangen, Germany

Aims: To perform the analysis of adverse events (AE) rate and trends of physiologically meaningful parameters in patients with cardiac implantable electronic devices (CIEDs) with the mobile remote monitoring option.

Methods: In 9 clinical centers of the Russian Federation and 2 clinical centers of the Republic of Kazakhstan, 126 patients with an implantable cardioverter-defibrillator (ICD) or a pacemaker (PM) equipped with the Home Monitoring (HM) technology (BIOTRONIK, Berlin, Germany) were enrolled. Based on the daily data transmission, all alarm alerts, all HM options changes and all AE were recorded with dated alert content and undertaken measures.

Results: The study patients, followed up at least for one year, experienced 42 adverse events (AE), of which 26 were serious AE (SAE) and 3 SAE were defined as device-related (SAED). ICD patients ( $=90)$ with concomitant coronary artery disease $(\mathrm{CAD})$ had a statistically significantly higher SAE prevalence $(\mathrm{p}=0.0249)$. Patients with CRT-D had a lower SAE rate than patients with dual- or single-chamber ICD $(\mathrm{p}=0.046)$. Downloads of Home Monitoring parameters for retrospective mathematical analysis were available for 60 ICD patients, of which 47 had episodes of ventricular tachycardia (VT), ventricular fibrillation (VF) and/or atrial tachyarrhythmia (AT). Machine learning analysis of the trends of the physiologically meaningful parameters revealed correlations between changes and arrhythmia episodes, with the random forest and gradient boosting methods demonstrating the random effect of the results.

Conclusion: Home Monitoring of CIED patients enables the evaluation of different devices applications and their clinical advantages. This might implement the prevention of adverse events and iatrogenic effects of pacing. Based on daily transmission of physiologically meaningful Home Monitoring parameters, the study results demonstrate the feasibility of developing a prediction algorithm for adverse events.

Key words: electrotherapy of heart; Home Monitoring; adverse events; trends of physiological parameters

Conflict of Interests: nothing to declare

Translated from Journal of Arrhythmology. 2019;26(2): 5-13; Original article submitted: 26.05 .2019

Corresponding author: Khassanov Ildar, E-mail: ildar.khassanov@biomed.uni-erlangen.de

For citation: Revishvili ASh, Lomidze NN, Abdrakhmanov AS, Nechepurenko AA, Ivanitsky EA, Belyaev OV, Popov SV, Lebedev DS, Lebedeva VK, Mikhailov SP, Pokushalov EA, Mamchur SE, Shugaev PL, Rekvava RR, Vasilyev SN, Kuptsov VV, Berdyshev VI, Sungatov RSh, Khassanov ISh on behalf of the ReHoming study investigators Remote monitoring for the early detection of changes in patient status using the Home Monitoring technology. Journal of Arrhythmology. 2019;26(2): 5-13; https://doi.org/10.35336/VA-2019-2-5-13.

Remote monitoring (RM) of patients with cardiac implantable electronic devices (CIEDs) has been in use for more than 20 years [1]. Currently, RM is recommended for patients with pacemakers (PMs), implantable cardioverter-defibrillators (ICDs) and systems for cardiac resynchronization therapy (CRT) as a part of standard follow-up (FU) strategies [2, 3].
Numerous clinical trials as well as randomized, have been performed with the Biotronik Home Monitoring (HM) systems. The TRUST study [4] has demonstrated statistically that relevant clinical events can be revealed in patients followed-up by RM much earlier than for patients, followed-up only by ambulatory visits. Clinical outcome can be improved 
through early diagnostics of important cardiac events, prone to serious complications (e.g. stroke as a consequence of persistent atrial fibrillation) [4-6], better patients' compliance [7] and satisfaction of therapy procedures [8].

On one hand, the COMPAS [6] and OEDIPE [9] clinical trials have shown that RM can significantly shorten time to necessary physician's intervention and, on the other hand, reduce greatly the number of unnecessary FUs, - approximately $50 \%$ of patients not needing a scheduled FU [10] as no changes in their therapy or their implant's program are necessary [11]. According to the "TRUST" [4] and "COMPAS" [6] trials, the related clinical burden can be reduced, both for physicians and patients, by $45 \%$ and $56 \%$, respectively.

The "ECOST" study showed [12] that HM can reduce the number of inadequate shocks by $52 \%$ and the number of related hospitalizations - by $72 \%$. The appropriate implant reprogramming enabled for reduction of the number of shock charges by $76 \%$, and of delivered shock discharges - by $71 \%$, with a significant positive effect on the battery longevity.

The most important clinical result have been reported in the "IN-TIME" trial [13], with a more than two-fold reduction of the overall and cardiovascular mortality in congestive heart failure (CHF) patients monitored remotely, in comparison to patients, followed-up only by scheduled ambulatory FUs.

The Russian Scientific Society of Arrhythmology, Electrophysiology and Cardiac Pacing has sponsored the "ReHoming" (Registry Home Monitoring) clinical trial with the goal to evaluate the clinical results of patient's remote monitoring FU in the Russian Federation and the Republic of Kazakhstan. The Home Monitoring generated alerts of relevant clinical events have been recorded in case report forms (CRF). Trends of physiologically meaningful parameters have been used by physicians to decide whether to intervene with an unscheduled FU. Serious adverse events including hospitalizations and cardiovascular complications have been recorded, as well as undertaken measures, like therapy or implant program changes.

\section{METHODS}

\section{Patient enrollment and study protocol}

Patients of both sexes older than 18 years were eligible to enroll in the study if they had an ICD or a PM with the mobile RM option (Home Monitoring BIOTRONIK, Germany). Exclusion criteria were:

- Post cardiac surgery or post infarction $<1$ month,

- More than two cardioversion shocks within last 6 months (for ICD patients),

- Lead dislodgement, and/or impedance, threshold, or sensing failure, loss of capture and inadequate ICD therapy,

- Implant-related infection,
- Inability to handle the "Home Monitoring" system correctly,

- Participation in another clinical study,

- Insufficient GSM coverage at patient's home,

- Pregnancy or nursing.

\section{Study design}

The Rehoming trial was designed as an open, multi-center and observational study. In addition to an ICD or a PM the enrolled patients received (or already had) a RM transmitting device (Cardiomessenger II-S, BIOTRONIK) with both the patient and the transmitter registered at the Home Monitoring Service Center (HMSC).

Regular patient's FUs were scheduled by the study investigators according to the guidelines intervals starting from the enrollment procedure: 3 months for ICD patients, and 6 months for PM patients (Fig. 1).

All FU results were documented in the patient's health record and then entered into the CRF. Russian Internet platform rehoming.dicoming.com was developed [14], with the downloaded Home Monitoring data, offering a possibility to fill in the CRF protocols online.

\section{Study parameters}

Continuous patient RM was aimed to register all alarming HM alerts with the "red" (urgent) and with the

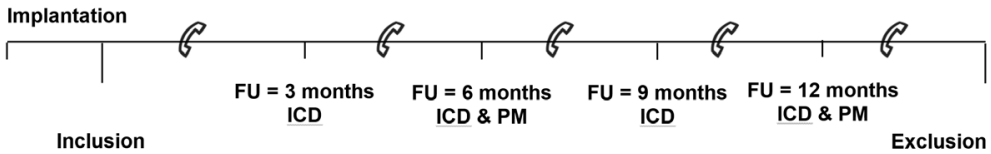

Figure 1. ReHoming study design. From the inclusion point the patient is followed up by "Home Monitoring". Besides regular ambulatory follow-ups (every 3 months for the ICD, every 6 months for the pacemaker) the investigator has to perform additional followup in the case of a relevant "Home Monitoring" alert, indicating a serious adverse event probability.

Table 1.

\section{Patient characteristics}

\begin{tabular}{|l|c|c|c|c|c|c|}
\hline \multirow{2}{*}{} & \multicolumn{2}{|c|}{$\mathrm{TP}(\mathrm{n}=119)$} & \multicolumn{2}{c|}{$\mathrm{ICD}(\mathrm{n}=89)$} & \multicolumn{2}{c|}{ PM (n=30) } \\
\cline { 2 - 7 } & $\%$ & $\mathrm{n}$ & $\%$ & $\mathrm{n}$ & $\%$ & $\mathrm{n}$ \\
\hline $\mathrm{AH}$ & $50.4 \%$ & 60 & $56.2 \%$ & 50 & $33.3 \%$ & 10 \\
\hline CAD & $51.3 \%$ & 61 & $52.8 \%$ & 47 & $46.7 \%$ & 14 \\
\hline VHD & $2.5 \%$ & 3 & $1.1 \%$ & 1 & $6.7 \%$ & 2 \\
\hline CMP & $10.9 \%$ & 13 & $9.0 \%$ & 8 & $16.7 \%$ & 5 \\
\hline DM & $47.9 \%$ & 57 & $62.9 \%$ & 56 & $3.3 \%$ & 1 \\
\hline HF Class I & $13.4 \%$ & 16 & $13.5 \%$ & 12 & $13.3 \%$ & 4 \\
\hline HF Class II & $28.6 \%$ & 34 & $25.8 \%$ & 23 & $36.7 \%$ & 11 \\
\hline HF Class III & $44.5 \%$ & 53 & $55.1 \%$ & 49 & $10.0 \%$ & 3 \\
\hline HF Class IV & $2.5 \%$ & 3 & $3.4 \%$ & 3 & $0.0 \%$ & 0 \\
\hline No HF & $3.4 \%$ & 4 & $4.5 \%$ & 4 & $0.0 \%$ & 0 \\
\hline Notinvestigated & $7.6 \%$ & 9 & $6.7 \%$ & 6 & & 4 \\
\hline Mean HF Class & \multicolumn{2}{|c|}{2.14} & \multicolumn{2}{|c|}{2.35} & & 1.43 \\
\hline
\end{tabular}

Here, and after on: TP - total population, ICD - implantable cardioverter-defibrillator population, $\mathrm{PM}$ - pacemaker population, $\mathrm{AH}$ - arterial hypertension, CAD - coronary artery disease, CHD - congenital heart disease, VHD - valvular heart disease, CMP - cardiomyopathy, DM diabetes mellitus, HF - heart failure. 
"yellow" (attention) status, and to register all resultant changes of the HM options. If necessary, based on the physician's decision and/or patient's need and in view of received HM alert, FU was scheduled, to prevent an anticipated a serious adverse event (SAE). An EchoCG was recommended in case of the following patient's status changes: dramatic increase of atrial arrhythmia burden, dramatic increase of the ventricular paced events ratio $\mathrm{Vp}$, rapid worsening of the Heart Failure Monitor (HFM, Biotronik) parameters.

Ambulatory FUs of patients with ICDs or CRT-D systems were scheduled 3, 6, 9 and 12 months after enrollment, and the following data were recorded:

- Implant printout of device parameters and statistical data (paroxysms of atrial tachyarrhythmia - AT, ventricular tachycardia - VT, mean ventricular rhythm, also, at rest, patient activity, number of ventricular extrasystoles (VES) per hour);

Table 2.

\section{Patient medication}

\begin{tabular}{|l|c|c|c|c|}
\hline \multirow{2}{*}{ Class I antiarrhythmics } & \multicolumn{2}{|c|}{$\mathrm{TP}(\mathrm{n}=119)$} & \multicolumn{2}{c|}{ ICD $(\mathrm{n}=89)$} \\
\cline { 2 - 5 } & $\%$ & $\mathrm{n}$ & $\%$ & $\mathrm{n}$ \\
\hline Beta blockers & 0.9 & 1 & 1.2 & 1 \\
\hline Class III antiarrhythmics & 59.5 & 66 & 70.6 & 60 \\
\hline Ca-antagonists & 8.1 & 9 & 4.7 & 4 \\
\hline Cardiac glycosides & 7.2 & 8 & 9.4 & 8 \\
\hline ACE inhibitors & 54.1 & 60 & 60.0 & 51 \\
\hline Diuretics & 56.8 & 63 & 64.7 & 55 \\
\hline Nitrovasodilators & 2.7 & 3 & 2.4 & 2 \\
\hline Anticoagulants & 46.0 & 51 & 43.5 & 37 \\
\hline Other & 64.9 & 72 & 61.2 & 52 \\
\hline On medication totally & 93.3 & 111 & 95.5 & 85 \\
\hline
\end{tabular}

Ventricular arrhythmias, documented in ICD and

Table 3. CRT-D patients as indication for the device implantation

\begin{tabular}{|c|c|c|c|c|c|}
\hline & \multicolumn{2}{|c|}{$\begin{array}{c}\text { ICD } \\
(n=59)\end{array}$} & \multicolumn{2}{|c|}{$\begin{array}{l}\text { CRT-D } \\
(n=30)\end{array}$} \\
\hline & & $\%$ & $\mathrm{n}$ & $\%$ & $\mathrm{n}$ \\
\hline \multicolumn{2}{|c|}{ Ventricular fibrillation (VF) } & 0.0 & 0 & 0.0 & 0 \\
\hline \multicolumn{2}{|c|}{ Ventricular tachycardia (VT) } & 42.4 & 25 & 16.7 & 5 \\
\hline \multirow{2}{*}{ VT type } & Monomorphic & 30.5 & 18 & 13.3 & 4 \\
\hline & Polymorphic & 11.9 & 7 & 3.3 & 1 \\
\hline \multirow{3}{*}{$\begin{array}{l}\text { Conscious- } \\
\text { ness loss }\end{array}$} & Pre-Syncope & 20.3 & 12 & 10.0 & 3 \\
\hline & Syncope & 3.4 & 2 & 0.0 & 0 \\
\hline & Clinical death & 5.1 & 3 & 0.0 & 0 \\
\hline \multicolumn{2}{|c|}{ Cryptogenic syncope } & 1.7 & 1 & 0.0 & 0 \\
\hline \multicolumn{2}{|c|}{ Primary prophylaxis of SCD } & 57.6 & 34 & 83.3 & 25 \\
\hline \multicolumn{2}{|c|}{ Secondary prophylaxis of SCD } & 42.4 & 25 & 16.7 & 5 \\
\hline \multicolumn{2}{|c|}{ Congestive heart failure } & 32.2 & 19 & 100 & 30 \\
\hline
\end{tabular}

Here and after on: CRT-D - cardiac resynchronization therapy with defibrillator population, SCD - sudden cardiac death.
- Necessity of the EchoCG procedure;

- Implant reprogramming;

- Changes of drug therapy;

- Events revealed by Home Monitoring.

\section{Statistical Analysis}

Study data were analyzed with the SAS software package (SAS Institute, USA), version 9.4. Mean value and standard square deviation (SD) are stated for parameter sets with normal distribution, median and quartiles for the other parameters. For categorical data, absolute and relative frequencies are given.

Metric study parameter sets were compared using the Student's t-test or the Wilcoxon-Mann-Whitney test (if the parametric test assumption was not fulfilled). Binary and categorical parameters were analyzed using the $\chi^{2}$-test and the Fisher exact test. Critical double-sided level of significance for all tests was 0.05 .

\section{Internet platform}

Besides the HMSC portal, investigators were offered to use the ReHoming portal [14] developed as a part of the universal HELTERBOOK ${ }^{\mathrm{TM}}$ [15] Internet platform. The portal enabled for comprehensive HM data mirroring and continuous study monitoring. Automatic options of statistical analysis were available with different filtering parameters, allowing analysis of study data in different patient sampling groups.

\section{Study endpoints}

The primary endpoint was the occurrence of a serious adverse event (SAE), including patient's death or hospitalization, complications from cardiovascular disease and the implanted device failure.

Secondary endpoints were the efficiency of the "Home Monitoring" technology to reveal the AEs and the clinical benefit of RM application within the country healthcare structure.

\section{Retrospective analysis of the Home Monitoring database}

To reveal possible correlation between the changes of the daily RM parameters and the probability of certain arrhythmia events, the trends of the physiologically meaningful parameters that could be used to develop a predictor of patient's status worsening were retrospectively analyzed [16]:

- Mean heart rate (HR) over 24 hours,

- HR at rest,

- Patients activity,

- VES per hour averaged over 24 hours,

- HR variability (HRV),

- Right ventricle lead impedance,

- Shock lead impedance,

- Ratio of atrial paced events Ap.

To evaluate the feasibility to develop a predictor algorithm, the events that are automatically recorded by Home Monitoring for ICD were chosen (i.e. episodes of AT, VT and ventricular fibrillation (VF)).

\section{RESULTS}

\section{Patient population}

In 9 research centers of the Russian Federation and 2 research center of the Republic of Kazakhstan, 126 patients were enrolled, 114 of which completed the trial and 12 - dropping out. The database contains the complete data 
of 119 patients, included in the study efficacy analysis population: 89 patients with ICDs, 30 with PM. Data for ICD patient were partly available and used for appropriate results analysis. Therefore, the total population size was 120 patients and the ICD population size was 90 patients.

For the total population of 119 patients, $88(73.9 \%)$ were male and, for the ICD population $(n=89), 69(77.5 \%)$ were male. Mean ages were $57.5 \pm 11.4$ and $56.8 \pm 11.4$, respectively. The main comorbidities were cardiovascular disease (mostly CHF) and diabetes mellitus (Table 1).

The majority of patients, $111(93.3 \%)$ and $85(95.5 \%)$ of the total and the ICD groups, respectively, received cardiovascular medications (Table 2). 59 patients of the total population (49.6\%) and 48 patients of the ICD population $(53.9 \%)$ were surgically treated due to coronary artery disease (CAD), congenital (CHD) or valvular heart disease (VHD), or arrhythmias. 30 patients of the ICD population $(n=89)$ had an ICD for cardiac resynchronization therapy (CRT-D systems).

Ventricular arrhythmia was the indication for the ICD or the CRT-D implantation in all ICD patients $(n=89)$, mostly for primary SCD prophylaxis - 59 patients, $66.3 \%$ (Table 3).

\section{Study safety analysis}

Safety analysis included 120 patients with a total 42 adverse events (AE), of which 4 were evidently related to the implantable device (AED) and 4 - possibly related. In total, 26 serious AE (SAE) were reported, of which 3 were SAED (related or probably related to the device). During the course of the study there were 2 patient deaths not related to the implant. The other $24 \mathrm{SAE}$ included patient hospitalizations due to different reasons: CHF worsening $(n=8,19.0 \%$ of all AE), VT $(n=7$, $17 \%), \operatorname{CAD}(n=2)$, gastritis $(n=2)$, acute myocardial infarction $(n=1)$, ischemic stroke $(n=1)$, permanent atrial fibrillation $(n=1)$, cardiac transplant rejection $(n=1)$ and lead dislodgement $(n=1)$.

Fisher exact double-sided test of ICD patients' data $(n=90)$ demonstrated statistically significant differences in SAE rate for patients with and without CAD, $p=0.0249$ (Table 4).

For some other cardiac diseases, no statistically significant difference were observed other than a trend for higher SAE rate was seen: for VHD - $p=0.1473$, and for diabetes mellitus (DM) $-\mathrm{p}=0.2151$.

Fisher exact double-sided test demonstrated a statistically significant lower SAE rate in patients with CRT-D than in patients with dual-chamber ICD, $\mathrm{p}=0.046$ (Table 4).

\section{Study efficacy analysis}

Upon CIED implantation and RM activation, 120 patients were followed-up according to the study protocol for an average of $28.3 \pm 10.1$ months ( $2 \div 43$ months). Mean annual number of HM messages was $43.6 \pm 35.6$ messages per year for the total population $(n=120),(5 \div 221)$. Home Monitoring of the ICD population $(n=90)$ documented ventricular arrhythmias in 52 patients: VF episodes - in 43, and VT - in 21 (Table 5).

Thirty four ICD patients $(n=89)$ received on the average $4[1 ; 11.5]$ defibrillation shocks per patient (maximum - 127), with an efficiency of $100[60.7 ; 100] \%$. Forty five $(50.6 \%)$ patients received anti-tachycardia pacing (ATP) therapy with an efficiency of $40[10.5 ; 78.1] \%$ in the VT zone $(n=29)$ and of $54.5[14.3 ; 99] \%$ in the VF zone $(n=30)$.

Of clinical interest was to reveal any correlation between the disease etiology and the rate of arrhythmias, registered by the implant. The most significant correlation in the ICD population $(n=90)$ was VT prevalence in patients with supraventricular tachycardia (SVT), $\mathrm{p}=0.0107$. VT episodes rates were statistically significantly lower in ICD patients with CHF in anamnesis lonely (Fisher test $\mathrm{p}=0.0320$ ), and with comorbidity of CHF and VHD, $\mathrm{p}=$ 0.0327 (Table 6). CAD did not increase in a statistically significantly manner the rate of VT detection, $\mathrm{p}=0.6706$.

\section{Clinical patient load}

According to the study protocol, investigators recorded different aspects of the clinical load related to patients FU and also based on VT presence (Table 7). The total number of patients' visits to clinic was 240 for an average of $0.97 \pm 0.56$ per patient per year. Medical care was requested by 41 patients. Patients with VT needed emergency help twice more often than patients without VT, while the mean number of hospitalization and unproductive days per year were approximately the same for both subgroups.

Clinical load was analyzed based on patients' etiology. Among the ICD population ( $\mathrm{n}=90)$, CHD patients created higher clinical load than VHD patients: days to first therapy - 213 and 354 days, unproductive days - 8.9 and 8.3 , hospitalization days -6.8 and 5.7, respectively. Efficacy of different ICD therapy types for CHD and VHD patients showed non significant statistical trends: ATP in VF zone $(56.6 \%$ vs $52.3 \%)$ and cardioversion shocks $(81.7 \%$ vs75.0\%) were more efficient in CHD patients, but ATP in

Table 4.

Serious adverse events (SAE) in ICD and CRT-D patients with and without $C A D$

\begin{tabular}{|l|c|c|c|}
\hline & SAE - Yes & SAE - No & Total \\
\hline CAD & 13 & 35 & 48 \\
\hline Yes & 3 & 39 & 42 \\
\hline No & 16 & 74 & 90 \\
\hline Total & 14 & 42 & 56 \\
\hline Implantable device type \\
\hline ICD & 2 & 28 & 30 \\
\hline CRT-D & 16 & 70 & 86 \\
\hline Total
\end{tabular}

Table 5.

Ventricular arrhythmias in ICD and CRT-D patients $(\boldsymbol{n}=90)$

\begin{tabular}{|c|c|c|c|c|}
\hline & \multicolumn{4}{|c|}{ Number of episodes } \\
\hline & ICD & & CRT-D & \\
\hline & Total (n) & Mean* & Total $(\mathrm{n})$ & Mean* \\
\hline VA & 52 & $10[2 ; 32.7]$ & 13 & $11[2 ; 30]$ \\
\hline VT & 21 & $9[2 ; 54]$ & 5 & $7[3 ; 24]$ \\
\hline VF & 43 & $7[2 ; 20.5]$ & 12 & $5.5[1 ; 16.2]$ \\
\hline PS & \multicolumn{2}{|c|}{$51(56.7 \%)$} & \multicolumn{2}{c|}{$13(14.4 \%)$} \\
\hline
\end{tabular}

* - per patient, VA - ventricular arrhythmias, PS - patient sample 
the VT zone was more efficient in VHD patients $(47.9 \%$ vs $41.9 \%$ ).

In accordance with the study protocol, investigators rated different aspects of the Home Monitoring technology for patients' $\mathrm{FU}$ with a 5-point scale (5 - highest rating). The mean ratings were: HMSC performance - 4.7, "traffic light" concept - 4.7, "IEGM online“ option - 4.7 and sufficiency of HM data - 4.6.

\section{Analysis of physiological parameters' trends}

For 60 ICD patients, long-term trends of daily recorded parameters were downloaded from the HMSC portal. Data for retrospective mathematical analysis were chosen according to the criteria of possible correlation analysis between the RM parameters' trends and the event onset probability. Therefore, the episodes with monitoring data available for at least 7 days and with no more than 2 successive blank days were selected. After trends review, 47 patients with events of the VT, VF and SVT type were selected. In order to build analytical models on the available dataset, we selected the following number of independent events: SVT - 200, VT - 27, VF - 38.

We used the cross validation technique to evaluate accuracy of possible correlations [17]:

- The dataset was randomly divided into five subgroups so that the number of records with and without the specific event were approximately similar in different subgroups;

- The data of four subgroups were used to determine

Table 6.

Rate of VT detection in ICD patients $(n=90)$ and comorbidities: SVT, CHF and VHD

\begin{tabular}{|l|c|c|c|}
\hline \multirow{2}{*}{} & \multicolumn{3}{|c|}{ VT detection } \\
\cline { 2 - 4 } & Yes & No & Total \\
\hline Supraventricular tachycardia \\
\hline Yes & 31 & 12 & 43 \\
\hline No & 21 & 26 & 47 \\
\hline Total & 52 & 38 & 90 \\
\hline Congestive HF & 23 & 26 & 49 \\
\hline Yes & 29 & 12 & 41 \\
\hline No & 52 & 38 & 90 \\
\hline Total & 23 & 19 & 42 \\
\hline Congestive HF \& valvular heart disease \\
\hline Yes & 33 & 15 & 48 \\
\hline No & 56 & 34 & 90 \\
\hline Total & \multicolumn{5}{|c}{} \\
\hline
\end{tabular}

which correlations (training sets) could predict the onset of a specific event;

- The models were validated on the retained data of the fifth subgroup.

To evaluate the models quality, a ROC-analysis (Receiver Operating Characteristic) was performed with the ROC_AUC (area under the curve) metrics [18]. The choice was due to the relatively low number of specific events in the dataset (VF, VT) and large time intervals with no events. The ROC_AUC metric does not depend on the number of specific events and, in general, reflects the ratio of truly classified cases of event occurring or not, with 1 meaning ideally correct prediction, and 0.5 - a random guess.

\section{Search for correlations and predictor modelling}

The search for correlations was performed with the following algorithms of machine learning:

- Decision tree - random forest classifier (search of parameters' values that could be the symptoms of the target event and splitting data trends in groups according to the parameters' values) [19];

- Support-vector networks with linear and radial kernels (values separation by hyperplanes in multi-dimensional space of parameters) [20];

- Nearest neighbors algorithm [21];

- Logistic regression (based on correlation of events and parameters) [22];

- Gradient boosting method [23].

These algorithms revealed significant deviations of ROC-curves from the diagonal demonstrating the possibility of the available parameters' set to predict specific events with a probability, significantly exceeding a random guess.

The best result for AT (with the largest dataset) was shown by the gradient boosting method with a ROC AUC $=0.79624, \min =0.73510$. Overall, owing to the relatively large number of samples, this event type was the best predicted. The following parameters were the most relevant for the modelled predictors (in order of importance): mean heart rate (HR), HR at rest, impedance of the right ventricular (RV) lead, mean number of ventricular extrasystoles (VES) per hour and patient activity.

Comparatively good intermediate results for VF (38 event samples) were achieved by the random forest (ROC $\mathrm{AUC}=0.71819, \min =0.55398)$ and gradient boosting methods (ROC_AUC $=0.66753, \min =0.53420576$ ). Even the worst case result was exceeding a random guess (though much weaker than for the AT event type). The

Clinical load for ICD patients in dependence on VT presence

Table 7.

\begin{tabular}{|l|c|c|c|}
\hline \multirow{2}{*}{} & \multicolumn{3}{|c|}{ VT detection } \\
\cline { 2 - 4 } & Yes $(\mathrm{n}=54)$ & No $(\mathrm{n}=36)$ & Total $(\mathrm{n}=90)$ \\
\hline Number of messages* & $17.3[11.2 ; 30.7]$ & $10.6[6.7 ; 14.5]$ & $14.9[8.4 ; 29.4]$ \\
\hline Days to first therapy & $199[61 ; 485]$ & - & $199[61 ; 485]$ \\
\hline Number of patients with inability days* & $12(22.2 \%)$ & $8(22.2 \%)$ & $20(22.2 \%)$ \\
\hline Number of patients with emergency help* & $9(16.7 \%)$ & $3(8.3 \%)$ & $12(13.3 \%)$ \\
\hline Days of hospitalization* & $0[0 ; 8.5]$ & $0[0 ; 7.9]$ & $0[0 ; 8.6]$ \\
\hline
\end{tabular}

* - per year 
most relevant parameters for the modelled predictor were as follow: PP-interval variability (HRV), RV-lead impedance, shock lead impedance, mean HR, number of high HRV intervals, ratio of cardiac cycles with atrial pacing Ap, patient activity, mean VES per hour and number of «mode switching» per day.

For the VT events (27 event samples), we were not able to define statistically reliable correlations though the result of the gradient boosting method were found to be slightly better than a random guess (ROC_AUC = $0.68984, \min =0.51504)$. The most relevant parameters for the modelled predictors were as follow: mean HR, $\mathrm{RV}$-lead impedance, presence of blanking monitoring data and mean atrial rate.

\section{DISCUSSION}

During the course of the ReHoming study we have developed a Russian portal to conduct remote follow-up of patients with CIEDs. As opposed to other similar portal, and in addition to the CIED compiled data, our portal also enables us to record other clinical data for their processing. The integrated automatic system for statistical analysis of the data allows for the processing of all recorded patients data as well as specific groups pooling according to different clinical feature of the investigator's choice (e.g. ICD or PM patients, CAD patients, etc.). These options give unlimited possibilities for research and management of clinical studies of any scale.

The newly developed study RM center has proven its efficacy and advantages that could be used in the future as an additional service to gather RM of patients of different clinics. It could be especially relevant for medical institutions with small number of patients, where additional personnel workload would be not cost-effective.

Strengthening the preventive aspect of this medical service is one of the priorities of healthcare development. Interventional arrhythmology and, especially CIED therapy, are currently leading innovative fields in the broad use of the RM technology. The "ReHoming" project is an example of the "Home Monitoring" technology localization in the healthcare structure that facilitates the development of guidelines for patients' FU with mobile RM. This clinical study demonstrates the potential of medical data integration and the machine learning methods for complex data analysis of large population cohorts to develop a predictor of patient's status worsening.

It must be outlined that the predictor models, presented in this paper use essentially nonlinear methods, and therefore, there is no direct linear relationship between the parameters' values and the probability of the event onset. Importantly, the implants record automatically both the parameters' trends and the predictable events, without any physician's or patient's intervention. This will become an important factor as the data volume will increase due to greater patient and physiological parameters number.

\section{STUDY LIMITATIONS}

Results of the ReHoming study are largely aligned with other studies on remote monitoring of CIED patients. However, our study is a registry with no control group for comparative analysis. It is advisable to organize a larger national trial including a control group in order to reveal the influence of the specific national healthcare system, and to verify statistically the clinical and economic advantages of CIED patients' remote monitoring.

\section{CONCLUSION}

Follow-up of the CIED patients with the RM technology enabled the evaluation of the clinical aspects of different implants' use and how to avoid iatrogenic pacing effects. The SAE rate was significantly lower in CRT-D patients than in patients with single- and dual-chamber ICDs.

Comparative analysis of the arrhythmias rate based on the diseases etiology showed statistically significant correlations between the VT number and SVT, CHF and VHD comorbidity.

Despite the limitations due to the small amount of statistical data, the study results demonstrate the possibility to develop a predictor of disease complications based on daily transmission of trends of physiologically meaningful parameters recorded by the implant. Machine learning algorithms, such as the random forest and gradient boosting methods, revealed results that were strongly exceeding a random guess.

The Internet portal developed in the context of the ReHoming project and the built-in automatic system of data statistical analysis provide a framework for the implementation of machine learning methods. CIED therapy has therefore become one of the clinically relevant fields of artificial intelligence development and application.

\section{ACKNOWLEDGEMENTS}

The ReHoming clinical study was organized in the frames of the scientific grant, awarded by BIOTRONIK SE \& Co. KG (Berlin, Germany).

Authors express their gratitude to Timur Zagidullin ("Atlant-Clinical" LLC, Moscow) for statistical analysis of the clinical study results.

\section{REFERENCES}

1. Hutten H, Schaldach M. Telecardiology Optimizing the diagnostic and therapeutic efficacy of the next implant generation. Progress in Biomedical Research. 1998; 3(1):1-4.

2. Dubner S, Auricchio A, Steinberg JS, et al. ISHNE/ EHRA expert consensus on remote monitoring of cardiovascular implantable electronic devices (CIEDs). Europace 2012; 14:278-293.

3. Slotwiner D, Varma N, Akar JG, et al. HRS Expert Consensus Statement on remote interrogation and monitoring for cardiovascular implantable electronic devices. Heart
Rhythm. 2015; 12 (7):e69-e100.

4. Varma N, Epstein AE, Irimpen A, et al for the TRUST Investigators. Efficacy and safety of automatic remote monitoring for implantable cardioverter-defibrillator follow-up: the Lumos-T safely reduces routine office device follow-up (TRUST) trial. Circulation. 2010; 122(4):325332.

5. Ricci RP, Morichelli L, Santini M. Home monitoring remote control of pacemaker and implantable cardioverter defibrillator patients in clinical practice: impact on medi- 
cal management and health-care resource utilization. Europace. 2008; 10 (2):164-170.

6. Mabo P, Victor F, Bazin P et al, on behalf of the COMPAS trial investigators. A randomized trial of long-term remote monitoring of pacemaker recipients (The COMPAS trial). Eur Heart J. (2011) doi: 10.1093/eurheartj/ehr419. First published online: November 29, 2011.

7. Varma N, Epstein AE, Irimpen A et al. Efficacy of calendar based ICD checks: conventional follow up compared to remote monitoring in the TRUST trial. European Heart Journal. 2009; 30 (abstract supplement): 278.

8. Ricci RP, Morichelli L, Quarta L et al. Long-term patient acceptance of and satisfaction with implanted device remote monitoring. Europace. 2010; 12(5):674-679.

9. Halimi F, Clémenty J, Attuel P et al. OEDIPE trial Investigators. Optimized post-operative surveillance of permanent pacemakers by home monitoring: the OEDIPE trial. Europace. 2008; 10:1392-1399.

10. Vardas PE, Auricchio A, Blanc JJ, et al. European Society of Cardiology; European Heart Rhythm Association. Guidelines for cardiac pacing and cardiac resynchronization therapy: the task force for cardiac pacing and cardiac resynchronization therapy of the European society of cardiology. Developed in collaboration with the European Heart Rhythm Association. Eur Heart J. 2007; 28:22562295 .

11. Heidbüchel H, Lioen P, Foulon S, et al. Potential role of remote monitoring for scheduled and unscheduled evaluations of patients with an implantable defibrillator. Europace. 2008 ; 10:351-357.

12. Kacet S, Guédon-Moreau L, Hermida J-S, et al. on behalf of the ECOST trial Investigators. Safety and effectiveness of implantable cardioverter defibrillator follow-up using remote monitoring: ECOST study. European Society of Cardiology, Paris, 29 August 2011 - http://www.escardio.org/congresses/esc-2011/congress-reports/Pages/7072-ECOST.aspx
13. Hindricks G, Taborsky M, Glikson $M$ et al. Implant-based multiparameter telemonitoring of patients with heart failure (IN-TIME): a randomised controlled trial. The Lancet. 2014; 384 (9943):583-590.

14. Revishvili ASh, Lomidze NN, Sungatov RSh, Khassanov ISh. Remote diagnosis and integration of the medical data for improvement of effectiveness of electrocardiotherapy. Journal of Arrhythmology 2016; 85:5-18 (in Russian). 15. Helterbook. State registration certificate of the computer program No. 2013661583. 11 December 2013 (Russian).

16. Sack $\mathrm{S}$, Wende ChM, Nägele $\mathrm{H}$ et al. Potential value of automated daily screening of cardiac resynchronization therapy defibrillator diagnostics for prediction of major cardiovascular events: Results from Home-CARE (Home Monitoring in Cardiac Resynchronization Therapy) study. European Journal of Heart Failure. 2011; 13:1019-1027.

17. Refaeilzadeh P, Tang L, Liu H. (2009) Cross-Validation. In: Liu L, Özsu MT (eds) Encyclopedia of Database Systems. Springer, Boston, MA.

18. Zweig MH, Campbell G. Receiver-operating characteristic (ROC) plots: a fundamental evaluation tool in clinical medicine. Clin Chem. 1993; 39:561-577.

19. Ho TK. The random subspace method for constructing decision forests. IEEE Transactions on Pattern Analysis and Machine Intelligence. 1998; 20(8):832-844.

20. Cortes C, Vapnik V. Support-vector networks. Machine Learning 1995; 20:273-297.

21. Altman NS. An introduction to kernel and nearest-neighbor nonparametric regression. The American Statistician. 1992; 46(3):175-185.

22. Aivazyan SA, Buchstaber VM, Yenukov IS, Meshalkin LD. Applied statistics: classification and decrease of dimensionality. M.: Finance and statistics; 1989. - 607 p. (in Russian).

23. Friedman J. Greedy Function Approximation: A Gradient Boosting Machine. - IMS 1999 Reitz Lecture. 